\title{
Assessment of Hypertension-Induced Deaths in Ghana: A Nation-Wide Study from 2012 to 2016
}

\author{
David Adedia1 ${ }^{*}$, Livingstone Asem², Simon Kojo Appiah³, Salifu Nanga1, Yeboah Boateng1, \\ Kwabena 0. Duedu4, Lotsi Anani ${ }^{5}$
}

\begin{abstract}
${ }^{1}$ Department of Basic Sciences, School of Basic \& Biomedical Sciences, University of Health and Allied Sciences, Ho, Ghana ${ }^{2}$ Department of Epidemiology \& Biostatistics, School of Public Health, University of Health and Allied Sciences, Ho, Ghana ${ }^{3}$ Department of Statistics and Actuarial Science, College of Science, Kwame Nkrumah University of Science and Technology, Kumasi, Ghana

${ }^{4}$ Department of Biomedical Sciences, School of Basic and Biomedical Sciences, University of Health and Allied Sciences, Ho, Ghana

${ }^{5}$ Department of Statistics and Actuarial Science, College of Basic and Applied Sciences, University of Ghana, Legon, Ghana Email: *dadedia@uhas.edu.gh, asemkal@yahoo.com, skappiah@knust.edu.gh,snanga@uhas.edu.gh, eyboateng@uhas.edu.gh, kduedu@uhas.edu.gh, alotsi@ug.edu.gh
\end{abstract}

How to cite this paper: Adedia, D., Asem, L., Appiah, S.K., Nanga, S., Boateng, Y., Duedu, K.O. and Anani, L. (2020) Assessment of Hypertension-Induced Deaths in Ghana: A Nation-Wide Study from 2012 to 2016. Journal of Data Analysis and Information Processing, 8, 158-170. https://doi.org/10.4236/jdaip.2020.83009

Received: May 30, 2020

Accepted: August 4, 2020

Published: August 7, 2020

Copyright $\odot 2020$ by author(s) and Scientific Research Publishing Inc. This work is licensed under the Creative Commons Attribution International License (CC BY 4.0).

http://creativecommons.org/licenses/by/4.0/ (c) (i) Open Access

\begin{abstract}
Globally, hypertension is one of the leading causes of death. It can potentially lead to heart disease and stroke, among others, that could result to premature death. In Ghana, hypertension is considered as a disease that contributes to an increase in outpatients' attendance. To assess the trend differentials of hypertension-induced deaths in Ghana, Chi-square test for equal proportions and Marascuilo procedure for pairwise comparison were performed using surveillance data on reported number of deaths from 2012 to 2016 across the then ten regions. The results show that incidence of hypertension-induced mortality was significantly different for almost all the regions and over the years. The incidence of hypertension-induced mortality has significantly reduced from 2012 to 2016. However, Volta Region recorded the highest incidence of mortality cases ( $p$-value less of 0.0001 ) than the other regions during the period under review, while the Upper East Region recorded continuous increase in incidence of mortality cases with the highest in 2016. The Eastern Region, Central Region, and Greater Accra Region recorded significantly ( $p$-value less of 0.0001 ) higher incidence of hypertension-induced mortality than the Ashanti Region, Brong Ahafo Region, Northern Region, Western Region and Upper West Region. The Upper West Region and Western Region had the lowest incidence of mortality. The decline in trend of hypertension-induced mortality could be attributed to some healthcare interventions put in place during the period. One of these interventions was the introduction of health insurance in 2003, a development which has been shown to af-
\end{abstract}


fect the health seeking behaviors of the people. It is, therefore, important to investigate factors affecting these spatial and temporal dynamics in order to determine appropriate ways to actively control the hypertension-induced deaths in the country. Public education on health should be intensified so as to totally curb hypertension and its attendant risks.

\section{Keywords}

Hypertension, Hypertension-Induced Mortality, Chi-Square Test, Marascuilo Test, Incidence Rates, Ghana

\section{Introduction}

Across the world, hypertension is said to be responsible for over 7.5 million deaths annually, which is about $12.8 \%$ of the total number of all deaths [1]. One in every three adults suffers from hypertension and about $51 \%$ of all deaths from stroke and $45 \%$ deaths from heart disease could be attributed to high hypertension alone [2] [3]. The estimated total number of people with hypertension in 2000 was 972 million, and this is projected to increase by $60 \%$ to a total of 1.56 billion by 2025 [4]. It is estimated that hypertension, also known as raised/high blood pressure, kills 9 million people every year [5] in the world.

In Ghana, the hypertension-induced proportional mortality rate is $5.2 \%$. As a matter of fact, hypertension is highly ranked among the top ten causes of mortality [6]. This is the situation elsewhere in the world. In Brazil, for instance, [7] reported that the main cause of mortality in that country was hypertension, as well as diabetes. These studies explained that deaths occurring in Brazil as a result of diabetes were actually due to the many risk factors associated with hypertension. Besides, the predisposing factors of hypertension were high, ultimately leading to the high hypertension-induced mortality.

A critical review of literature indicates that prior to 1930, hypertension was virtually nonexistent in African countries although the disease was thought to be common in Europe and in North America at the time. However, by 1950, post mortem reports suggested high blood pressure was beginning to be a problem in Sub-Saharan Africa. From 1961, it has actually been found to be a public health concern in Sub-Saharan Africa [8]. The prevalence of hypertension is now the highest in Africa, within the range 15\% - 70\%, of adults aged 25 and above, while the high-income countries have a lower prevalence of hypertension [9]. The rising prevalence of hypertension is attributed to a number of factors, including the growing population, ageing and behavioural risk factors, such as unhealthy diet, alcohol, lack of physical activity, tobacco use, obesity, high cholesterol, diabetes mellitus and persistent stress [10] [11], according to the Ministry of Health (MOH) [12] report on non-communicable diseases, between $19 \%$ and $40 \%$ of all adults in Ghana had hypertension. Also, the number of reported new cases of hypertension in outpatient public health facilities increased more than ten-fold from 49,087 in 
1988 to 505,180 in 2007 . Over the same period, hypertension relative to the total reported outpatient diseases increased from $1.7 \%$ to $4.0 \%$ in all ages.

Hypertension has continuously ranked in the top five outpatient diseases for more than 15 years in Ghana, accounting for 3\% - 5\% of all new outpatient diseases across all ages. In 2002, hypertension accounted for $8.9 \%$ of institutional deaths (excluding teaching hospitals), compared with malaria which accounted for $17.1 \%$ of the deaths (Ghana Health service (GHS) [13]). At the same time, Ghana's Ministry of Health reports have also listed hypertension as the leading cause of deaths in over 45-year-olds [8] in the country. In 2008, cardiovascular diseases caused about $14.5 \%$ of institutional deaths which was more than $13.4 \%$ of deaths due to malaria. Moreover, hypertension was highly ranked among the cardiovascular diseases [14]. In addition, Darkobonsu [15] reported that hypertension was among the top ten diseases causing mortality in the Sekondi-Takoradi, while [16] also revealed that $19.4 \%$ of maternal deaths in the southern part of Ghana were due to hypertension. Clearly, hypertension-related cases are on the ascendency. In this study, statistical assessment of deaths due to hypertension in all the then 10 regions of Ghana was performed. This sought to establish the trend differentials in incidence of mortality rates in the regions (Greater Accra (GAR), Eastern (ER), Western (WR), Volta (VR), Ashanti (AR), Brong Ahafo (BAR), Northern (NR), Upper East (UER), and Upper West (UWR) Regions) as well as over the years (2012-2016).

\section{Materials and Methods}

Data on hypertension-induced mortality in Ghana was collected from the District Health Information Management System (DHIMS-2) at the Ho Regional node. DHIMS-2 was adopted by Ghana as a district web-based health information management platform to collect, collate and report on public health surveillance data. The Regional population and growth rates recorded for 2010 census by Ghana Statistical Service [17] were used to estimate the population for each of the ten regions using geometric progression approach [18]. The estimated population and mortality cases were then used to determine the actual population at risk, as well as incidence rates of hypertension-induced mortality. In order to assess the changes in the incidence of mortality due to hypertension in Ghana from 2012 to 2016, chi-square test of equality of proportions was used. Usually, to compare more than two population means, the one-way analysis of variance (ANOVA) is used for normal quantitative data whilst Kruskal-Wallis test is used for non-normal or ranked data. The chi-square test of goodness of fit (for proportions being the mortality incidence rates) is used because the data (proportions) are categorized. The objective of the chi-square test is to test whether the $k$ independent sample proportions which are the incidence rates $\left(p_{i} ; i=1,2 \ldots, k\right)$ are the same. That is, the null hypothesis that the incidence of mortalities for the various $(k)$ regions in Ghana (or over the years) are the same $\left(p_{1}=p_{2}=\cdots=p_{k}\right)$ is tested against the alternative hypothe- 
sis that at least two of the population incidence of mortalities are different $\left(p_{i} \neq p_{j}\right)$, where $i \neq j=1,2, \cdots, k$ [19]. According to Williams [19], the chi-square test statistic is given by

$$
\chi^{2}=\sum_{i=1}^{k} n_{i} \frac{\left(p_{i}-p\right)^{2}}{v}
$$

where $p_{i}=\frac{r_{i}}{n_{i}}, \quad r=\sum_{i=1}^{k} r_{i}, \quad n=\sum_{i=1}^{k} n_{i}, \quad p=\frac{r}{n}, \quad v=\frac{n p(1-p)}{n-1}, \quad r_{i}$ is the number of people who died as a result of hypertension, and $n_{i}$ is the population at risk of hypertension-induced mortality in each region or year.

\section{The Pairwise Multiple Comparison}

After testing the significance difference in $k$ sample proportions, pairwise tests were performed to test the hypotheses: $H_{0}: p_{i}=p_{j}$ (incidence of mortality rate in one region is the same as the other) verses $H_{1}: p_{i} \neq p_{j}$ (incidence of mortality in one region differs from the other, where $i \neq j$ using Marasscuilo method for multiple comparisons. The Marascuilo procedure for multiple comparisons is used as a post-hoc analysis after chi-square test of equality of proportions show significant differences. The tables for Marascuilo test were arranged in the order of ranks of incidence of hypertension-induced mortality where the least incidence had the least rank and the highest incidence had the highest rank. Groups were identified by alphabets. Years or Regions with representation of the same alphabet signifies a non-significant difference between them and with different alphabets representation signifying a significant difference.

\section{Results}

The incidence of hypertension-induced mortality was estimated with the denominator as the estimated population at risk for the region or year and it was used to determine the differences in mortality at each location (region) and time (year). Figure 1 is a line graph presenting the yearly distribution of the incidence of hypertension-induced mortality in Ghana from 2012 to 2016. Overall, mortality decreased from $3.7 \%$ in 2012 to $2.4 \%$ in 2016 (Figure 1). The decrease in mortality was similar for the GAR and BAR, but different for the rest. The VR, $\mathrm{CR}, \mathrm{NR}$ and UWR showed increase in the first three years before showing consistent decrease. The AR showed a decrease from 2012 to 2013 but increase from 2013 to 2014 followed by a decrease in the incidence of mortality from 2014 to 2016 (Figure 2) in that order. The UER had mortality rising from 2012 to 2016, whereas the UWR and WR were relatively stable over the period. The VR had the highest incidence of mortality followed by CR and AR, then GAR, UER, AR, BAR, NR, WR and UWR (Figure 2). The incidence for the UWR was, however, the lowest, ranging from 0.012 to 0.018 .

Table 1 presents the results of chi-square test, which is used to test whether the sample incidence of mortalities are the same for all the years. As the computed $p$-value is far less than the significance level, $\alpha=0.01(<0.0001)$, the null 


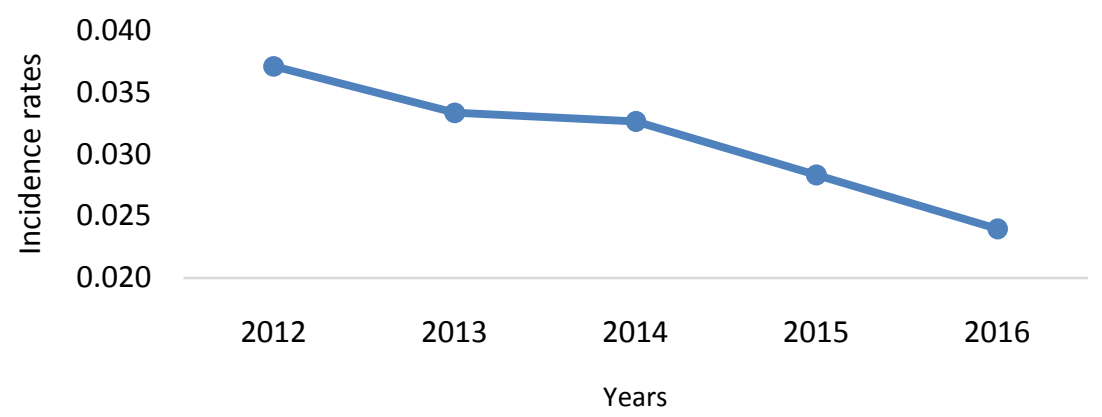

Figure 1. Yearly distribution of incidence of hypertension-induced mortality in Ghana.

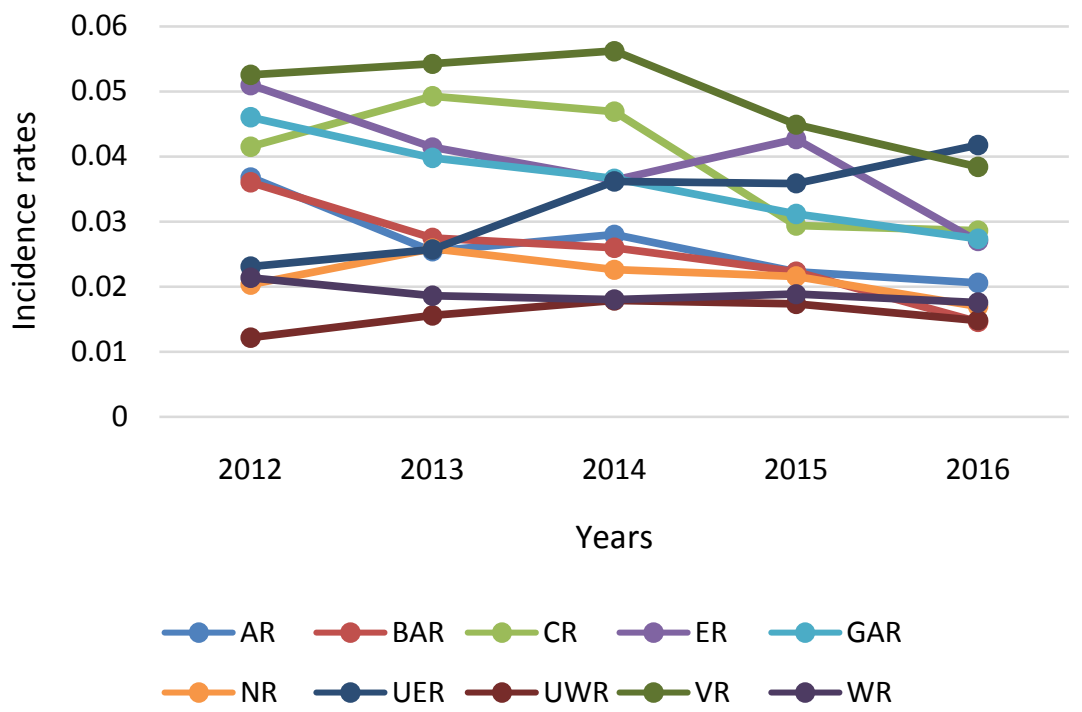

Figure 2. The regional yearly distribution of incidence of hypertension-induced mortality in Ghana.

Table 1. Chi-square test for differences in incidence of hypertension-induced mortalities in Ghana (from 2012 to 2016).

\begin{tabular}{cc}
\hline Chi-square (Observed value) & $87,060.5871$ \\
Chi-square (Critical value) & 9.4877 \\
DF & 4 \\
$p$-value & $<0.0001$ \\
Alpha & 0.05 \\
\hline
\end{tabular}

hypothesis that the incidences of hypertension-induced mortality are the same for all years is rejected, and can therefore be concluded that the incidences of mortalities are not the same. The Marascuilo procedure in Table 2 presents the multiple comparison procedure which was further used to identify the years that were significantly different from each other in terms of incidence of hypertension-induced mortality. The test revealed that there is a significant difference in incidence of mortality due to hypertension in the five years (Table 2). The hypertension-induced mortality reduced systematically from 2012 to 2016. 
Table 2. Multiple comparisons of incidences using the Marascuilo procedure (from 2012 to 2016).

\begin{tabular}{cccccc}
\hline Sample & Proportion & \multicolumn{3}{c}{ Groups } \\
\hline 2016 & 0.0240 & A & & & \\
2015 & 0.0283 & & B & & \\
2014 & 0.0327 & & C & & \\
2013 & 0.0334 & & D & \\
2012 & 0.0372 & & & E \\
\hline
\end{tabular}

Table 2 presents pairwise difference of the incidence rates of mortality due to hypertension among the ten regions of Ghana for year groups. Tables 3-7 present the results of chi-square tests for equality of incidence of mortality in all regions for 2012, 2013, 2014, 2015 and 2016, respectively. The $p$-values $(<0.0001)$ for these omnibus chi-square tests showed significant differences in the incidences of mortality for all regions (Tables 3-7). The probabilities of committing type I error in all the omnibus chi-square tests in this study were less than $0.01 \%$. This implies that there is a lower risk of wrongly rejecting the null hypotheses of no difference in incidences of hypertension-induced mortality.

The multiple comparisons showed that there is a significant difference in incidence of the mortality rates due to hypertension in the ten regions in 2012 (Table 8). The mortality rates from the least to the highest based on regions for 2012 are: UWR, NR, WR, UER, BAR, AR, CR, GAR, ER and VR. This means the UWR reported the least incidence of mortality and VR recorded the highest. In 2013, the incidence of mortality occurred in ascending order as UWR, WR, AR, UER, NR, BAR, GAR, ER, CR and VR (Table 9). The UWR recorded the least incidence rate of mortality and VR recorded the highest as in 2012. However, AR, UER and NR were ranked "C", implying that the incidence of mortality in AR were significantly different from all the other regions except UER and NR. In 2014, UWR followed by WR were ranked "A", which means incidence of mortality was the same in the two regions but they both had the least significant incidence of mortality as compared to other regions (Table 10). The UER, ER and GAR reported similar incidence of hypertension-induced mortality and the records in the 3 regions were significantly lower than CR and VR but higher than the rest. In 2015, AR and BAR were ranked "D", implying they reported similar incidences of mortality, but significantly higher incidence of mortality than NR, WR and UWR, and significantly lower incidence of mortality than CR, GAR, UER, ER and VR (Table 11). The incidence of hypertension-induced mortality for 2015 in ascending order is as: UWR, WR, NR, AR, BAR, CR, GAR, UER, ER and VR. The VR recorded the highest incidence of mortality in 2015. The results for 2016 showed that all regions reported significantly different incidence of mortality, with BAR and UWR reporting the least and UER reporting the highest (Table 12). The ER and GAR reported significantly lower incidence of mortality than UER, VR and CR but higher than the rest. The incidence of 
Table 3. Chi-square test for differences in incidence of hypertension-induced mortalities for the Regions in Ghana for 2012.

\begin{tabular}{cc}
\hline Chi-square (Observed value) & $97,120.7985$ \\
Chi-square (Critical value) & 16.9190 \\
DF & 9 \\
$p$-value & $<0.0001$ \\
alpha & 0.05 \\
\hline
\end{tabular}

Table 4. Chi-square test for differences in incidence of hypertension-induced mortalities in Ghana for 2013.

\begin{tabular}{cc}
\hline Chi-square (Observed value) & $101,056.5548$ \\
Chi-square (Critical value) & 16.9190 \\
DF & 9 \\
$p$-value & $<0.0001$ \\
alpha & 0.05 \\
\hline
\end{tabular}

Table 5. Chi-square test for differences in incidence of hypertension-induced mortalities for the Regions in Ghana for 2014.

\begin{tabular}{cc}
\hline Chi-square (Observed value) & $92,698.7867$ \\
Chi-square (Critical value) & 16.9190 \\
DF & 9 \\
$p$-value & $<0.0001$ \\
alpha & 0.05 \\
\hline
\end{tabular}

Table 6. Chi-square test for differences in incidence of hypertension-induced mortalities for the Regions in Ghana for 2015.

\begin{tabular}{cc} 
Chi-square (Observed value) & $69,037.8984$ \\
Chi-square (Critical value) & 16.9190 \\
DF & 9 \\
$p$-value & $<0.0001$ \\
alpha & 0.05 \\
\hline
\end{tabular}

Table 7. Chi-square test for differences in incidence of hypertension-induced mortalities for the Regions in Ghana for 2016.

\begin{tabular}{cc}
\hline Chi-square (Observed value) & $60,870.1412$ \\
Chi-square (Critical value) & 16.9190 \\
DF & 9 \\
$p$-value & $<0.0001$ \\
alpha & 0.05 \\
\hline
\end{tabular}


Table 8. Multiple comparisons of incidence of hypertension-induced mortalities for the Regions in Ghana using the Marascuilo procedure for 2012.

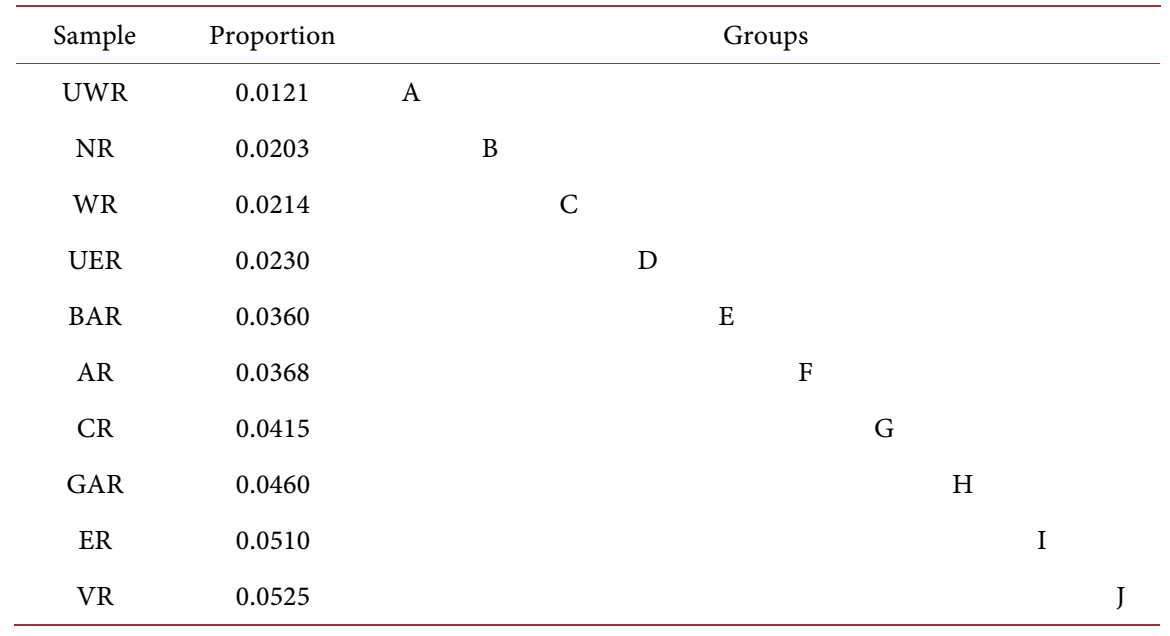

Table 9. Multiple comparisons of incidence of hypertension-induced mortalities for the Regions in Ghana using the Marascuilo procedure for 2013.

\begin{tabular}{ccccccc}
\hline Sample & Proportion & & \multicolumn{3}{c}{ Groups } \\
\hline UWR & 0.0156 & A & & & & \\
WR & 0.0186 & & B & & & \\
AR & 0.0255 & & C & & & \\
UER & 0.0257 & C & & & \\
NR & 0.0258 & C & & & & \\
BAR & 0.0275 & & D & & & \\
GAR & 0.0398 & & & E & & \\
ER & 0.0414 & & & & F & \\
CR & 0.0493 & & & & & G \\
VR & 0.0542 & & & & & \\
\hline
\end{tabular}

Table 10. Multiple comparisons of incidence of hypertension-induced mortalities for the Regions in Ghana using the Marascuilo procedure for 2014.

\begin{tabular}{|c|c|c|c|c|c|c|c|c|}
\hline Sample & Proportion & & & & Groups & & & \\
\hline UWR & 0.0179 & A & & & & & & \\
\hline WR & 0.0180 & A & & & & & & \\
\hline NR & 0.0226 & & B & & & & & \\
\hline BAR & 0.0260 & & & $\mathrm{C}$ & & & & \\
\hline $\mathrm{AR}$ & 0.0280 & & & & $\mathrm{D}$ & & & \\
\hline UER & 0.0361 & & & & & E & & \\
\hline ER & 0.0364 & & & & & $\mathrm{E}$ & & \\
\hline GAR & 0.0366 & & & & & $\mathrm{E}$ & & \\
\hline CR & 0.0469 & & & & & & 1 & \\
\hline VR & 0.0562 & & & & & & & G \\
\hline
\end{tabular}


Table 11. Multiple comparisons of incidence of hypertension-induced mortalities for the Regions in Ghana using the Marascuilo procedure for 2015.

\begin{tabular}{cccccccc}
\hline Sample & Proportion & \multicolumn{5}{c}{ Groups } \\
\hline UWR & 0.0173 & A & & & & & \\
WR & 0.0188 & & B & & & & \\
NR & 0.0215 & & & C & & & \\
AR & 0.0223 & & & D & & & \\
BAR & 0.0223 & & D & & & \\
CR & 0.0294 & & & E & & \\
GAR & 0.0312 & & & & F & & \\
UER & 0.0358 & & & & & G & \\
ER & 0.0427 & & & & & & H \\
VR & 0.0449 & & & & & & \\
\hline
\end{tabular}

Table 12. Multiple comparisons of incidence of hypertension-induced mortalities for the Regions in Ghana using the Marascuilo procedure for 2016.

\begin{tabular}{|c|c|c|c|c|c|c|c|c|c|}
\hline Sample & Proportion & \multicolumn{8}{|c|}{ Groups } \\
\hline BAR & 0.0146 & A & & & & & & & \\
\hline UWR & 0.0148 & A & & & & & & & \\
\hline NR & 0.0169 & & B & & & & & & \\
\hline WR & 0.0175 & & & $\mathrm{C}$ & & & & & \\
\hline $\mathrm{AR}$ & 0.0206 & & & & D & & & & \\
\hline ER & 0.0270 & & & & & E & & & \\
\hline GAR & 0.0273 & & & & & E & & & \\
\hline CR & 0.0286 & & & & & & $\mathrm{~F}$ & & \\
\hline VR & 0.0384 & & & & & & & G & \\
\hline UER & 0.0417 & & & & & & & & $\mathrm{H}$ \\
\hline
\end{tabular}

mortality are ranked in ascending order by regions as: BAR, UWR, NR, WR, AR, ER, GAR, CR, VR and UER.

Table 4 contains the results of chi-square test for differences in incidence of hypertension-induced mortalities for the Regions in Ghana in 2013.

\section{Discussion}

This study was carried out to assess the differences in incidence of hypertension-induced deaths in the then ten regions of Ghana (BAR, UWR, NR, WR, AR, ER, GAR, CR, VR and UER). It was revealed that the incidence of hypertension-induced deaths has been decreasing steadily from the year 2012 to 2016 . This development is probably due to the positive effects of some interventions and programs that affect health seeking-behavior like the introduction of the National Health Insurance Scheme (NHIS) [20] [21]. The programs such as the 
NHIS, healthy life lifestyles, health promotion and treatments are very effective to lower the risk of hypertension in Ghana [20]. The effectiveness of the NHIS in helping control hypertension has been highlighted by [20], who made it clear that even the very poor communities also suffer from hypertension, thus, the NHIS aids their access to life-saving drugs and care.

Although the three northern regions were considered the poorest [22] [23] [6], the general decrease in rates was not the same for all of them. Although the intervention programs could be responsible for the decrease in the hypertension-induced deaths over the years, this was not the case for UER. Using the 2014 Ghana Demographic and Health Survey data, [24] assessed the risk factors of hypertension in Ghanaian men and concluded that coming from UER and ER were related to family history of hypertension. The authors stated that the high prevalence of hypertension in these regions cannot be easily explained. The reasons why hypertension increased steadily in the UER, thus, could be multifaceted. These may include low attendance at hospital and poverty as indicated by other health indicator reports and studies [6] [24].

A study by [24] reported that men with higher economic status and educational background are more likely to have history of hypertension. [25] also stated that people with the highest grade of employment are more likely to be hypertensive. Similarly, adult wealth was positively associated with hypertension. People with the least literacy level and economic status in the UER, as well as with low awareness of the condition may account for lifestyles which predispose some of the inhabitants to hypertension. However, the steady increase in the incidence of hypertension-induced mortality in the UER needs further assessment.

In terms of the availability of healthcare, NR, UER and UWR recorded the fewest health facilities and hospital beds in Ghana, from 2011 to 2014 [26]. However, these regions also had the lowest cases of new reported outpatient hypertension with UWR recording the lowest of them [6]. One possible reason for this could be the fact that the fewest health facilities and Hospital beds recorded by the UWR could account for the likely less access to the health facilities by people from this region, which then leads to having lower incidence of hypertension-induced mortality.

Although the rates within the UWR, NR and the WR did not decrease as happened for the other regions, they should constitute the lowest. Other studies have reported low prevalence of hypertension in the WR [15]. The high hypertension-induced mortality in some of regions like the VR, CR, ER and GAR could be as a result of lifestyle and other risk factors of hypertension [20] [27].

\section{Conclusions}

The incidence of hypertension-induced deaths in Ghana differs among the regions and has been decreasing steadily over the years (2012-2016). VR recorded the highest incidence of mortality cases than other regions. ER, CR, GAR, and UER recorded significantly higher incidence of hypertension-induced mortality 
than the AR, BAR, NR, WR and UWR. The UWR and WR had the lowest incidence of mortality. The results suggest that there may be some health care interventions or indicators that may have contributed to the decrease in prevalence of hypertension-induced mortality over the years. One of these could be the introduction of health insurance in 2003, which has been shown to affect health seeking behaviors of the populace [21]. It is, therefore, important to investigate the factors affecting the dynamics in order to determine appropriate ways to actively control hypertension-induced deaths.

Based on the findings of this study, we suggest that the Public Health Department of the Ghana Health Service should partner with the media to sensitize the citizens on the early signs and symptoms of hypertension. Also, there is the need for people to go in for periodic checkups. In the case of those diagnosed, they must constantly take their medications. The Public Health Department of the Ghana Health Service should also educate the citizens on the importance of living a healthy life style in order to reduce hypertension and its risks.

\section{Limitations}

The difficulty in having access to data on some variables such as the demographics of subjects limited the study and therefore may limit our inferences toward important markers such as age and gender as well as complete intervention activities. We recommend a further study be conducted taking into consideration age, gender and lifestyle of the study population.

\section{Data Availability}

The data for this study is available.

\section{Funding Statement}

The study was funded by contributions by individual authors.

\section{Contributions}

All authors contributed to this manuscript and approve its submission. DA, SN and SKA conceived the problem and the research design whilst DA, SN, SKA, $\mathrm{LA}, \mathrm{YB}, \mathrm{KOD}$ and $\mathrm{AL}$ were actively involved in the collection of the data and analysis. All the authors helped in the draft and also reviewed the final manuscript.

\section{Acknowledgements}

We thank the $\mathrm{MoH}$, Ghana for making available the data for this study. We also thank Daniel Abaye and Nathaniel Glover-Meni (UHAS, Ho, Ghana) for helpful review comments during the preparation of this manuscript.

\section{Conflicts of Interest}

There are no competing interests. 


\section{References}

[1] Wilson, F.H., Disse-Nicodeme, S., Choate, K.A., Ishikawa, K., Nelson-Williams, C., Desitter, I., Feely, M.P., et al. (2001) Human Hypertension Caused by Mutations in WNK Kinases. Science, 293, 1107-1112. https://doi.org/10.1126/science.1062844

[2] World Health Organization (2020) New Data Highlight Increases in Hypertension, Diabetes Incidence.

https://www.who.int/mediacentre/news/releases/2012/world health statistics 2012 0516/en

[3] World Health Organization (2013) A Global Brief on Hypertension: Silent Killer, Global Public Health Crisis: World Health Day 2013 (No. WHO/DCO/WHD/2013.2). World Health Organization. https://doi.org/10.5005/ijopmr-24-1-2

[4] Damasceno, A., Azevedo, A., Silva-Matos, C., Prista, A., Diogo, D. and Lunet, N. (2009) Hypertension Prevalence, Awareness, Treatment, and Control in Mozambique. Hypertension, 54, 77-83. https://doi.org/10.1161/HYPERTENSIONAHA.109.132423

[5] Qureshi, A.I., Suri, F.K., Kirmani, J.F. and Divani, A.A. (2005) Prevalence and Trends of Prehypertension and Hypertension in United States: National Health a Nutrition Examination Surveys 1976 to 2000. Medical Science Monitor, 11, CR403-CR409.

[6] Ghana Health Service (2015) The Health Sector in Ghana: Facts and Figures 2015.

[7] Francesli, A., Reis, N., Lima, J.C., Beccaria, L.M., Ribeiro, D.F. and Cesarino, C.B. (2015) Hypertension and Diabetes-Related Morbidity and Mortality Trends in a Municipality in the Countryside of São Paulo. Revista Latino-Americana de Enfermagem, 23, 1157-1164. https://doi.org/10.1590/0104-1169.0533.2661

[8] Biritwum, R.B., Amoah, A.G.B. and Pobee, J.O.M. (2006) Cardiovascular Diseases in Sub-Saharan Africa. ASCOM Publishers.

[9] Ataklte, F., Erqou, S., Kaptoge, S., Taye, B., Echouffo-tcheugui, J.B., Kengne, A.P., Taye, B., et al. (2014) Burden of Undiagnosed Hypertension in Sub-Saharan Africa: A Systematic Review and Meta-Analysis. American Heart Association, Dallas. https://doi.org/10.1161/HYPERTENSIONAHA.114.04394

[10] Addo, J., Smeeth, L. and Leon, D.A. (2007) Prevalence, Detection, Management, and Control of Hypertension in Ghanaian Civil Servants. Ethnicity \& Disease, 18, 505-511.

[11] de Lima Santos, P.C.J., de Oliveira Alvim, R., Ferreira, N.E., de Sá Cunha, R., Krieger, J.E., Mill, J.G. and Pereira, A.C. (2011) Ethnicity and Arterial Stiffness in Brazil. American Journal of Hypertension, 24, 278-284. https://doi.org/10.1038/ajh.2010.244

[12] Ministry of Health, Ghana (2012) National Policy for the Prevention and Control of Chronic Noncommunicable Diseases in Ghana.

[13] Ghana Health Service (2012) The Health Sector in Ghana: Facts and Figures.

[14] Ghana Health Service (2009) The Health Sector in Ghana: Facts and Figures 2009.

[15] Darkobonsu, H. (2015) The Major Death Causing Diseases in Ghana (A Case Study of Sekondi Takoradi Metropolis). International Journal of Scientific and Innovative Mathematical Research, 3, 36-49.

[16] Der, E.M., Moyer, C., Gyasi, R.K., Akosa, A.B., Tettey, Y., Akakpo, P.K., Anim, J.T., et al. (2013) Pregnancy Related Causes of Deaths in Ghana: A 5-Year Retrospective Study. Ghana Medical Journal, 47, 158.

[17] Ghana Statistical Service (2012) Population \& Housing Census: Summary Report of 
Final Results.

[18] Modi, M.A. (2017) Population Forecasting for Design of Dhrafad Regional Water Supply System, Gujarat. International Journal for Innovative Research in Science \& Technology, 4, 2349-6010.

[19] Williams, D.A. (1988) Tests for Differences between Several Small Proportions. Journal of the Royal Statistical Society: Series C (Applied Statistics), 37, 421-434. https://doi.org/10.2307/2347316

[20] Bosu, W.K. (2010) Epidemic of Hypertension in Ghana: A Systematic Review. BMC Public Health, 10, Article No. 14. https://doi.org/10.1186/1471-2458-10-418

[21] Blanchet, N.J., Fink, G. and Osei-Akoto, I. (2012) The Effect of Ghana's National Health Insurance Scheme on Healthcare Utilisation. Ghana Medical Journal, 46, 76-84.

[22] Ghana Statistical Service (2015) Ghana Poverty Mapping Report.

[23] Cooke, E., Hague, S. and McKay, A. (2016) The Ghana Poverty and Inequality Report: Using the 6th Ghana Living Standards Survey 2016.

[24] Nyarko, S.H., Osei, E., Komesuor, J. and Ananga, M.K. (2017) Prevalence and Predictors of Hypertension History among Ghanaian Men. Ghana Journal of Geography, 9, 50-63.

[25] Addo, J., Smeeth, L., Leon, D.A., Addo, J., Smeeth, L. and Leon, D.A. (2009) Socioeconomic Position and Hypertension: A Study of Urban Civil Servants in Ghana. Journal of Epidemiology and Community Health, BMJ Publishing Group, 63, 646-650. https://doi.org/10.1136/jech.2008.081828

[26] Ghana Health Service (2015) Ghana Health Service 2014 Annual Report.

[27] Owusu, I.K., Boadi, R.K., Afari-Kissi, A., Adu-Boakye, Y. and Adu, Y. (2015) Logistics Regression Analysis of Risk Factors Associated with Hypertension in Kumasi Metropolis, Ghana. Open Science Journal of Clinical Medicine, 3, 205-211. 